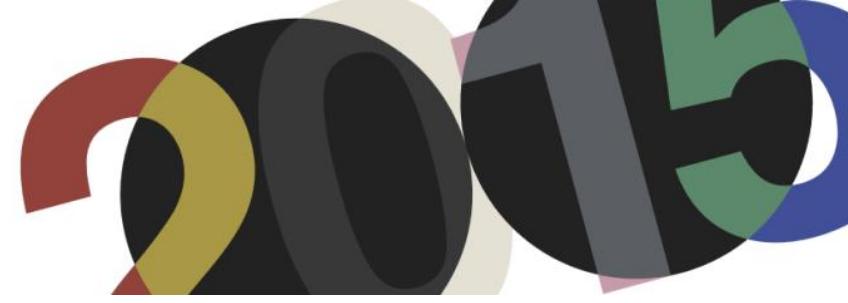

DOI: http://dx.doi.org/10.4995/LC2015.2015.630

\title{
F VAL. Feria Valencia y Guillermo Jullian de la Fuente. L'héritage de Le Corbusier
}

\author{
Federico Carro Gil*, Miguel Navarro Pérez**, Marta Mompó García*** \\ *Universidad Politécnica de Madrid/PhD Escuela de Arquitectura de A Coruña \\ **Universidad Politécnica de Madrid \\ ***Universidad Politécnica de Valencia
}

Resumen: La Mezquita de Córdoba, el paradigma del Mat-Building y la invitación al juego, están detrás de una obra cuya pertenencia a la órbita lecorbuseriana debe ser plenamente reconocida: la Feria de Valencia (1967) de Guillermo Jullian de la Fuente.

Cincuenta años después de la muerte de Le Corbusier y desaparecido, también, quién en los últimos años de vida del maestro fue su principal colaborador, apenas queda nada de la única obra de los herederos del Atelier de la rue de Sèvres construida en España.

Tomando como idea el paradigma de "juego" pretendemos, con nuestro estudio, avanzar en el conocimiento de esta obra para encontrar su vinculación con ese modo de entender lo arquitectónico que Le Corbusier nos propone cuando afirma que, reunidos bajo la luz, de modo sabio, correcto y magnífico, los volúmenes (las formas arquitectónicas) juegan.

Abstract: The Mosque of Cordoba, the paradigm of Mat-Building and the invitation to the game, are behind a work whose membership lecorbuseriana orbit should be fully recognized: the Feria de Valencia (1967) of Guillermo Jullian de la Fuente. Fifty years after the death of Le Corbusier, and Guillermo dead too, who in the last years of the life of the Master was his main collaborator, there is nothing left of the unique work of the heirs of the Atelier de la rue de Sèvres built in Spain.

On the idea to the paradigm of "play " we intend, in our study, to advance in the knowledge of this work to find its links to this way of understanding the architecture that Le Corbusier proposed to us when he states that, gathered under the light, so wise, correct and magnificent, the volumes (the architectural forms) play.

Palabras clave: Juego; Atelier; Venecia; Valencia.

Keywords: Game; Atelier; Venice; Valencia.

\section{Introducción}

Tratar de interpretar la Feria de Valencia al margen de la figura de Le Corbusier constituye un ejercicio ciertamente contradictorio ya que, con la misma seguridad con la que se puede afirmar que el proyecto no es del maestro, se debe afirmar que la Feria de Valencia es obra lecorbuseriana. Es más, de todos los proyectos que hace cincuenta años quedaron huérfanos sobre las mesas de dibujo del Atelier de la rue de Sèvres, la Feria de Valencia fue el que -con el impulso creativo de los herederos de aquel taller- consiguió recibir el testigo del ejercicio magistral de Charles-Edouard Jeanneret.

Sobre la base de esta idea, el objetivo de nuestro trabajo no se orienta pues a mediar en la controversia sobre la posible vinculación del maestro con el proyecto para Valencia. Nuestra intención pasa, sobre todo, por reorientar el debate dentro de sus auténticos límites: los que sitúan a la Feria de Valencia como verdadera obra del taller de Le Corbusier. 
Si fue por casualidad, o no, cómo es que llegó a levantarse en Valencia una edificación tan directamente relacionada con el Atelier del Corbu, es algo que no afecta al fondo de esta investigación. Si fue por casualidad, fue sin duda un feliz suceso. Si, por el contrario, hubo la intención de vincular el proyecto del recinto ferial valenciano a los herederos espirituales de Le Corbusier, fue un feliz acierto. Pero ni una cosa ni la otra añaden o restan valor arquitectónico a los pabellones que en 1968 comenzaron a construirse a las afueras de Valencia siguiendo el proyecto del arquitecto chileno Guillermo Jullian de la Fuente.

La concreción con la que queremos abordar este estudio nos lleva a estructurar el trabajo en tres apartados concatenados. En el primero de ellos, unos apuntes sobre la figura de Jullian nos conducirán a la formulación de un paradigma directamente relacionado con esa tendencia al orden y a la articulación, que está en la esencia del proceso creativo de la proyectación arquitectónica: nos referimos a la idea de "juego".

A la luz de este paradigma nos acercaremos al proyecto de la Feria de Valencia para proponer -en un segundo apartado de nuestro estudio- su lectura en relación con una obra con la que comparte, no sólo rasgos de una misma arquitectura, sino también, y sobre todo, el participar de ese espíritu de juego que anima y explica gran parte de la obra lecorbuseriana. Nos referimos al proyecto del Hospital de Venecia cuya continuación, tras la muerte del maestro, se solapa en el tiempo con el proyecto para Valencia. Sólo una indagación orientada por esta idea nos permitirá establecer los vínculos entre ambas arquitecturas, más allá de las evidencias.

Cerraremos el estudio volviendo la vista atrás para indagar en el nacimiento de este proyecto, poner orden en los datos fragmentados que nos son conocidos, con el propósito de completar una base lo suficientemente firme en la que sustentar un juicio crítico sobre esta obra.

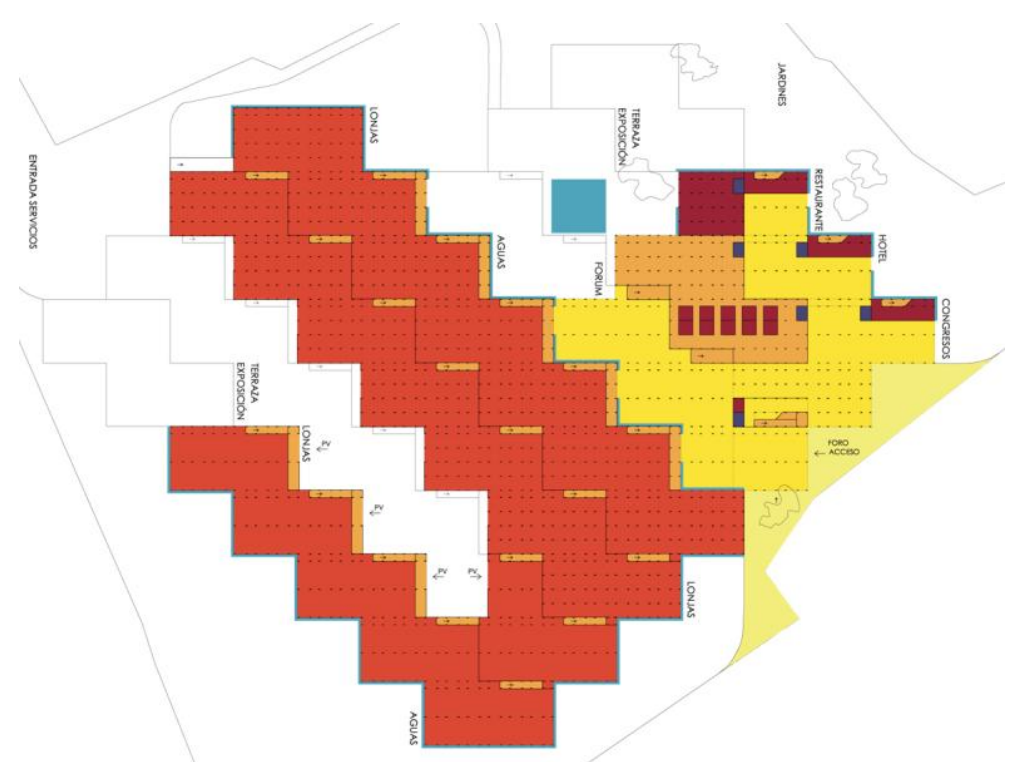

1. Plano canónico de la Feria de Valencia.

\section{El juego como paradigma}

Guillermo Jullian de la Fuente, nace en 1931 en Valparaíso (Chile) y se forma como arquitecto en la Escuela de Arquitectura de la Universidad Católica de esa ciudad. En 1958, apenas finalizada su formación académica, se embarca rumbo a Europa con el propósito de conocer a Le Corbusier y trabajar con él. Después de un tiempo visitando gran parte de su obra construida en el continente -una suerte de "peregrinaje" lecorbuseriano- en enero 
de 1959 escribe al maestro, solicitando formalmente un puesto en el Atelier: "Je viens vous demander si vous pouviez m'accepter comme un disciple de plus pour collaborer dans votre ouvre qui est déjà historie"1.

La respuesta, aunque negativa, dejaba abierta la puerta a colaborar de alguna manera. Poco después, una breve carta del maestro - esta vez en agradecimiento a unos apuntes de la Plaza de San Marcos de Venecia que Jullian le había hecho llegar- contiene una advertencia que, para un amante de la arquitectura como sin duda lo era Jullian, no estaba exenta de ironía y sarcasmo: "Le coup de crayón est une chose dangereuse chez les architectes" $"$.

No obstante la participación en la obra lecorbuseriana -si bien fuera del Atelier- da comienzo pronto, al entrar a trabajar para la ingeniería que estaba desarrollando el proyecto del Estadio de Bagdad. Y así, antes de finalizar el verano de 1959, una invitación a almorzar en casa del Corbu va a señalar el inicio de una colaboración que se prolongará a lo largo de seis intensos años, hasta la muerte del maestro.

Al evocar el ambiente que Jullian descubre en el laboratorio de Le Corbusier, recurre a una doble metáfora en la que se condensa un modo muy personal de entender el trabajo junto al maestro: "el atelier era una especie de caja de Pandora donde estaban todas las ideas de Le Corbusier, sus libros, sus viajes, sus obras, todo lo cual usábamos como base para trabajar nuevos proyectos. Todas las cosas que utilizábamos para trabajar estaban ahí, desde antes, nosotros jugábamos con ellas"3.

Esta alegoría a la creatividad del juego, entendido como la esperanza que ha quedado encerrada en la caja de Pandora, la queremos convertir en el hilo conductor del análisis de la Feria de Valencia. Jullian -como nos recuerda el profesor Claudio Vásquez en su introducción al número monográfico que la revista Massilia le dedica en 2007- al abandonar el Atelier de la rue de Sèvres era portador del tesoro de haber conocido la manera de trabajar de un maestro que, en muchos aspectos, había reformulado la arquitectura del siglo XX. Un modo de trabajar en consonancia con aquel universo poético que formaba parte de sus vivencias en la Escuela de Valparaíso ${ }^{4}$.

Para establecer el sentido que queremos imprimir al paradigma en torno al que va a girar el análisis de la Feria de Valencia, vamos a detenernos brevemente en el ensayo de Johan Huizinga Homo ludens, publicado por primera vez en 1938.

En esta obra, Huizinga aborda la realidad y el significado del juego como fenómeno cultural. Para el autor de este fundamental ensayo sobre la naturaleza lúdica de la cultura, la primera caracterización del juego tiene que ver con la libertad: "Todo juego es, antes que nada una actividad libre".

\footnotetext{
${ }^{1}$ La carta enviada por Jullian a Le Corbusier se encuentra publicada en el "Anexo 1" de Massilia 2007.

${ }^{2}$ La respuesta del Corbu (fechada el 2 de marzo de 1959) esta publicada en: Pérez de Arce, Rodrigo. Guillermo Jullian. Obra abierta. p. 207. En esta misma obra se reproducen además los bocetos enviados por Jullian a Le Corbusier (p. 15).

${ }^{3}$ Jullian de la Fuente citado por: Vásquez, Claudio. "Conversación con Guillermo Jullian de la Fuente”. En Massilia 2007. p. 18. Esta referencia al juego y la caja de Pandora se encuentra también (expresada en términos muy parecidos) en la entrevista que Amedeo Petrilli le hace al arquitecto en 1998; publicada en: Petrilli, Amedeo. Il testamento di Le Corbusier: il progetto per l'Ospedale di Venezia. p. 120.

${ }^{4}$ A este respecto recomendamos la lectura del artículo de Pérez Oyarzún "Valparaíso y los años formativos", publicado en: Massilia 2007.

${ }^{5}$ Huizinga, Johan. Homo ludens. p. 20.
} 
Pero a esta primera característica, el juego incorpora, para Huizinga, otras relacionadas con ese alejamiento de la vida "corriente", ese "estar encerrado en sí mismo" -que supone el jugar- para refugiarse en una esfera de actividad que posee su propia tendencia. Un universo que determina, para el juego, sus límites de tiempo y de espacio, y dentro del cual el juego es orden: crea orden.

De este modo, esta aspiración del juego hacia el orden y la articulación, dibuja los límites de un paradigma interpretativo del hecho arquitectónico que trasciende el plano metafórico en el que el concepto suele quedar encerrado. Un paradigma que sirve así para interpretar el sentido que Jullian imprime a su propio modo de acercarse al proyecto. No es extraño, pues, que la añoranza del juego, modele su memoria cuando afirma: "El Atelier de la rue de Sèvres... siempre fue un lugar alegre porque Le Corbusier trabajaba como un niño"6.

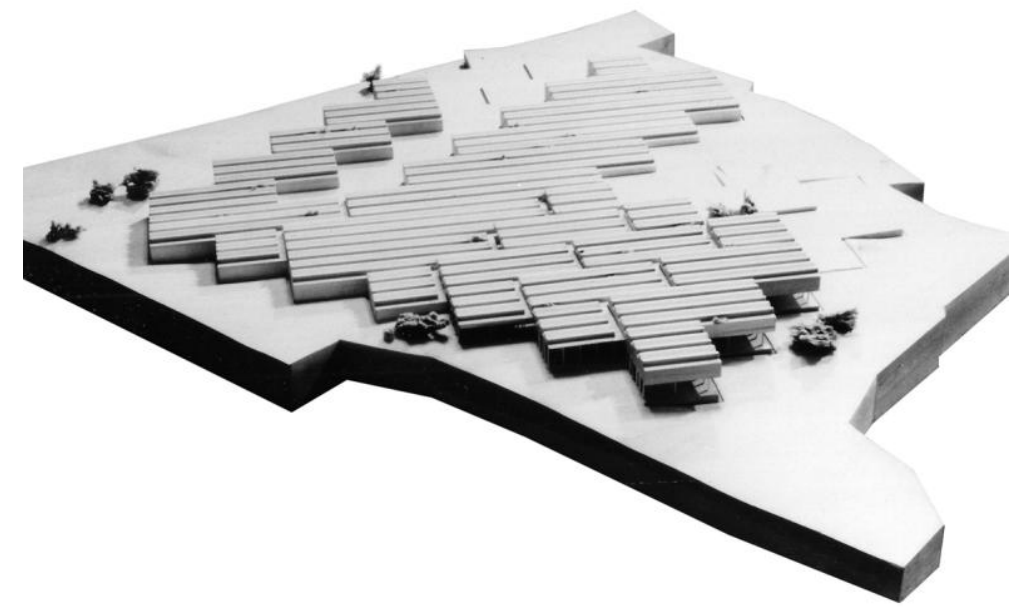

2. Vista de la Maqueta de Feria de Valencia.

\section{La esvástica y el dominó quebrado}

En el análisis de la Feria de Valencia, las referencias a la Gran Mezquita cordobesa así como el ejemplo del MatBuilding, constituyen acotaciones que, a pesar de haber sido abordadas en diferentes comentarios sobre el proyecto, deben sin embargo ser consideradas como parte de su interpretación.

A Córdoba -aunque de pasada- se refiere Jullian en la entrevista que Amedeo Petrilli le realiza en $1998^{7}$. En ella viene a señalar ese deseo de construir un espacio interpretado a partir de un orden de pilares -los pilotis lecorbuserianos- que, junto al manejo de la luz cenital, protagonizan el proyecto para Valencia. Asimismo la Mezquita, cuya grandiosa arquitectura estaba, muy probablemente, entre las intenciones del proyecto de la Feria, aparece como imagen iluminadora en un momento de la entrevista que, en 2006, le hace María Celia O’Byrne: "así es que [sic] está pensada la Feria de Valencia".

\footnotetext{
${ }^{6}$ Jullian de la Fuente citado por: Pérez de Arce, Rodrigo. Op. cit. p. 41.

${ }^{7}$ Petrilli, Amedeo. Op. cit. p. 121.

${ }^{8}$ O'Byrne Orozco, María Cecilia. "Entrevista a Guillermo Jullian de la Fuente". En: El proyecto para el Hospital de Venecia de Le Corbusier. Director: Josep Quetglas. Universidad Politécnica de Cataluña - Escuela Técnica Superior de Arquitectura de Barcelona, 2007. Cuaderno V. p. 65.
} 
Por otra parte, el concepto de "Mat" -cuya traslación al español (estera) se queda lejos del significado arquitectónico alcanzado por el término inglés- constituye una referencia de carácter general a un tipo de edificación cuyas conexiones con la Feria de Valencia resultan evidentes.

En efecto, el Mat, expresaba una aspiración de la arquitectura moderna -y más concretamente de la arquitectura que estaba en discusión dentro del seno del Team 10- por alcanzar e interpretar ese "anónimo colectivo" en el que -como señala Alison Smithson en su transcendental ensayo sobre el significado y la caracterización del MatBuilding- las funciones venían a enriquecer lo construido y el individuo adquiría nuevas libertades de actuación favorecidas por un orden nuevo y cambiante, basado en la interconexión, los patrones de asociación compactos y las posibilidades de crecimiento, disminución y cambio ${ }^{9}$.

Aunque el concepto de Mat-Building aparece plenamente formulado casi una década después del proyecto de Jullian para la Feria de Valencia, la idea -como señala Smithson- fue tomando forma progresivamente y moldeándose a través de diferentes proyectos de los que el de la Universidad Libre de Berlín (1963), del equipo Candilis-Josic-Woods, terminará convirtiéndose -con su construcción- en el ejemplo paradigmático ${ }^{10}$.

Entre las arquitecturas que componen la mirada retrospectiva de Smithson sobre el Mat, aparece un proyecto que va a convertirse en el marco de referencia en el que podemos situar la Feria de Valencia: la propuesta de 1964 de Le Corbusier y Jullian para el Hospital de Venecia.

En efecto, la Feria de Valencia de Jullian de la Fuente, cuya gestación tiene lugar durante el desarrollo -ya sin Le Corbusier- del proyecto del Hospital de Venecia, mantiene con éste muchas similitudes: el grafismo, el manejo de la forma arquitectónica en la consecución de una edificación de carácter horizontal, el carácter secundario de las fachadas frente a la importancia del plano de cubierta y la solución concreta a la iluminación de los espacios interiores, señalan estos puntos en común. Pero más allá de estas circunstancias, es el espíritu de "juego" el que anima ambos proyectos. Si en Venecia, Jullian y Le Corbusier juegan con la rotación de las unités de soins en la conformación de una esvástica que resuelve la circulación horizontal dentro del edificio, en Valencia esas mismas piezas - convertidas ahora en lonjas- construyen una suerte de dominó quebrado, magistralmente adaptado a la topografía, cuya vocación es la de extenderse sobre el territorio desplegándose como un gigantesco mosaico.

Esvástica y dominó quebrado se convierten así en elementales mecanismos compositivos que explican ambos proyectos. Mecanismos en los que se encierran las claves para la creación de la obra. De este modo, al apoyándonos en la idea de "juego", el estudio se reorienta hacia la descomposición analítica del proyecto. Una descomposición que nos permite llegar, no sólo al conocimiento de los elementos que integran la totalidad, entendidos como principio constituyente de la forma arquitectónica, es decir: como origen y parte de ésta, sino también, y sobre todo, a la comprensión de las relaciones de orden que mantienen dentro de la misma.

Recorreremos, pues, un camino para ir de los hechos a la ley que los rige, tratando de expresar la constitución de la forma arquitectónica como síntesis de un proceso creador y unificador. El paradigma de juego será nuestra

\footnotetext{
${ }^{9}$ Smithson, Alison. "How to Recognise and Read Mat-Building: Mainstream Architecture as It Has Developed towards the Mat-Building”. En: Sarkis, Hashim (Ed.). Le Corbusier's Venice Hospital and the Mat Building Revival. p. 91 . El artículo de Alison Smithson se publicó por primera vez en septiembre de 1974 en la revista Architectural Design.

${ }^{10}$ Ibid.
} 
apoyatura, y los maravillosos diagramas funcionales de la Feria de Valencia, publicados por Jullian en 1968 en la revista Lotus -a los que más adelante nos referiremos- indican que no vamos desencaminados.

\subsection{Venecia}

En Venecia, Le Corbusier y Jullian acometen la proyectación de un complejo orden horizontal, consecuente con la tipología y la trama de la ciudad, que implicaba la creación de una densa masa de edificación atravesada por patios. En la consecución de este orden, la creación de una unidad básica de edificación -la unité de bâtisseconstituye el germen del proyecto.

Esta parte elemental del conjunto se proyecta a partir del nivel superior de la edificación: la planta de las habitaciones - cellule- de los enfermos, que se va a convertir en el plano canónico del Hospital.

La unité de bâtisse conforma un cuadrado en el que desde un núcleo central -campiello- que conecta en vertical las distintas plantas del edificio, parten cuatro calles cuya disposición en esvástica imprime un orden rotacional a la unidad. Sobre este orden se va a apoyar la pieza secundaria del juego: la unidad de cuidados -unité de soinsque organiza la disposición de las habitaciones en torno a una serie de pasillos.

A través de la unité de bâtisse, circulación y función quedan dirigidas por un mismo engranaje -el de la rotaciónque termina por imponer sus reglas de juego a las unidades contiguas y, por extensión, a toda la planta.

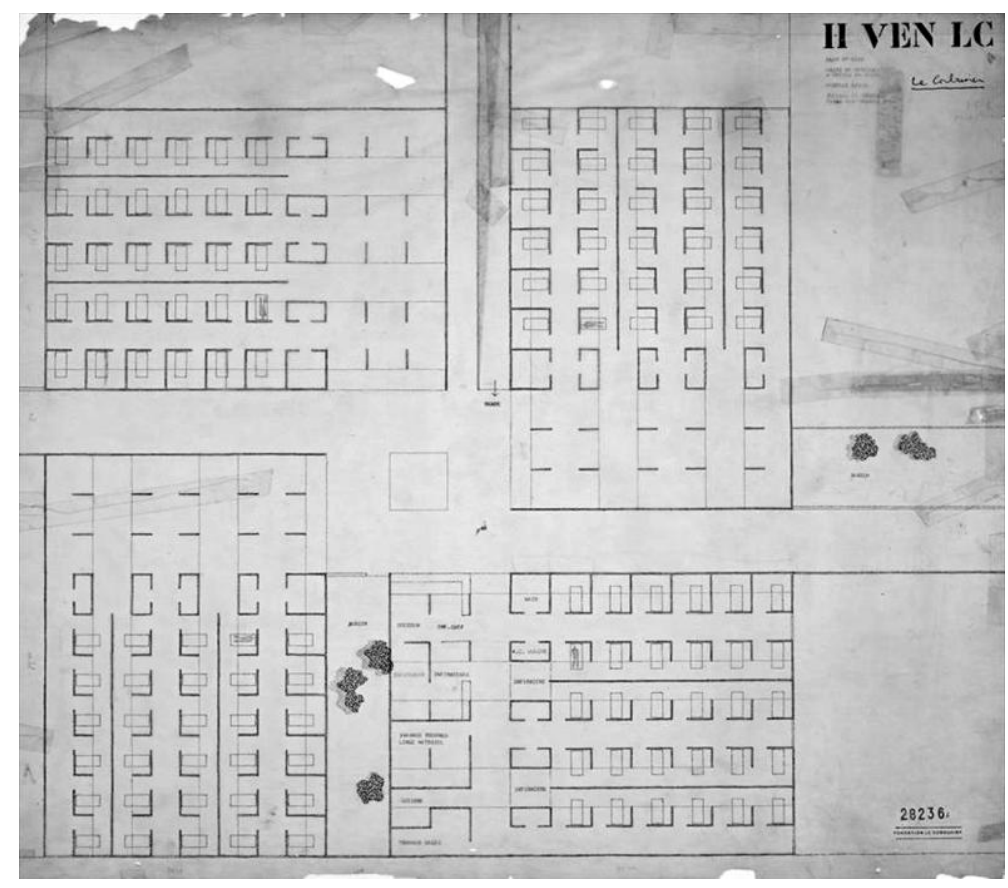

3. H VEN LC. Plano No 6286: "Unité de bâtisse - 4 Unités de soins" (escala 1:100, 1/10/1964) CFLC-ADAGP. 
En un ensayo aparecido por primera vez en 1966 en Architectural Design, Alan Colquhoun hace un análisis de dos de los últimos proyectos de Le Corbusier -el edificio de la Embajada de Francia en Brasilia y el Hospital de Venecia- en el que muy acertadamente examina las coincidencias entre ambas arquitecturas. Para Colquhoum, a pesar de las diferencias funcionales y organizativas entre una y otra propuesta, existe la evocación de una "respuesta compleja" en la que se superponen diversos niveles, pero en la que el recurso a un esquema fijo hace posible la descomposición analítica de su arquitectura. De este modo, la forma no se piensa como algo que emerge de la función, sino como algo basado en "esquemas ideales", y es en ese nivel en el que, para este autor, las funciones, libremente desplegadas, entablan con la forma un diálogo repleto de incidencias estéticas inesperadas ${ }^{11}$. Su conclusión nos aporta una idea que estimamos clave para entender la conexión de estos proyectos -en los que de manera tan decisiva intervino Jullian de la Fuente- con el proyecto de la Feria de Valencia: "la impresión de complejidad, en Venecia, como en Brasilia, es el resultado del impacto de varios subsistemas heterogéneos sobre unos esquemas básicos que, en sí mismos, son extremadamente simples ${ }^{12}$.

Como veremos, la elementalidad del juego en esvástica que explica el orden complejo del Hospital de Venecia, se traducirá en un elemental juego en dominó quebrado que revela el orden complejo de la Feria de Valencia.

\subsection{Valencia}

En Valencia el juego de elementos que componen la unidad básica del proyecto se simplifica. Ésta ya no es tanto una pieza de piezas - como era el caso de la unité de bâtisse del Hospital de Venecia- como una unidad construida a partir de un módulo básico de edificación: la lonja.

Pero para entender el significado de este elemento del proyecto se debe prestar atención a una serie de láminas que constituyen el primer escalón conceptual del mismo. Nos referimos a un conjunto de diagramas que, bajo el título de "programa", fueron desarrollados por Jullian en colaboración con José Oubrerie y Fernando Domeyko $^{13}$.

Se trata de un conjunto de dibujos fechados en París el 15 de febrero de 1967 en los que se investiga a cerca de la creación de una topología para el proyecto. El punto de partida para esta indagación lo constituyen los diferentes sistemas circulatorios a los que tiene que dar respuesta el conjunto de la Feria. En un primer grado de aproximación a la geometría del recorrido, la traza de éste se apoya en una retícula de tres por tres módulos cuadrados sobre la que se pueden dibujar diferentes circuitos construidos a partir del giro a 90 grados.

Con estos diagramas, Jullian parece ir un paso más allá de la creación de una simple traza lineal, reinterpretando la "grilla" y orientando la búsqueda a la consecución de una auténtica "textura". El entramado del proyecto va adquiriendo cada vez mayor complejidad, mediante operaciones de superposición de las circulaciones y la división y combinación de los diferentes sectores en los que se puede organizar el conjunto. De ahí que el último de estos diagramas se refiera, precisamente, a la creación de una "matriz" capaz de dar respuesta a la implantación de la Feria sobre un territorio todavía abstracto.

\footnotetext{
${ }^{11}$ Colquhoun, Alan. "Interacciones formales y funcionales. Un estudio de dos de los últimos proyectos de Le Corbusier". En: Arquitectura moderna y cambio histórico: ensayos 1962-1976. p. 43. En ambos proyectos fue decisiva la participación de Jullian de la Fuente, hasta el punto de continuar con los trabajos tras la muerte de Le Corbusier.

${ }^{12}$ Ibid.

${ }^{13}$ Estas láminas fueron publicadas en la revista Lotus. № 5. 1968. pp. 36-37.
} 
Un segundo escalón conceptual, da comienzo cuando ese entramado básico de la matriz se superpone a la topografía del sitio, dando lugar a lo que, el profesor Rodrigo Pérez de Arce, interpreta como verdadero "campo de juego" ${ }^{\prime 14}$. Surgiendo de este modo la necesidad de definir la pieza esencial del mismo.

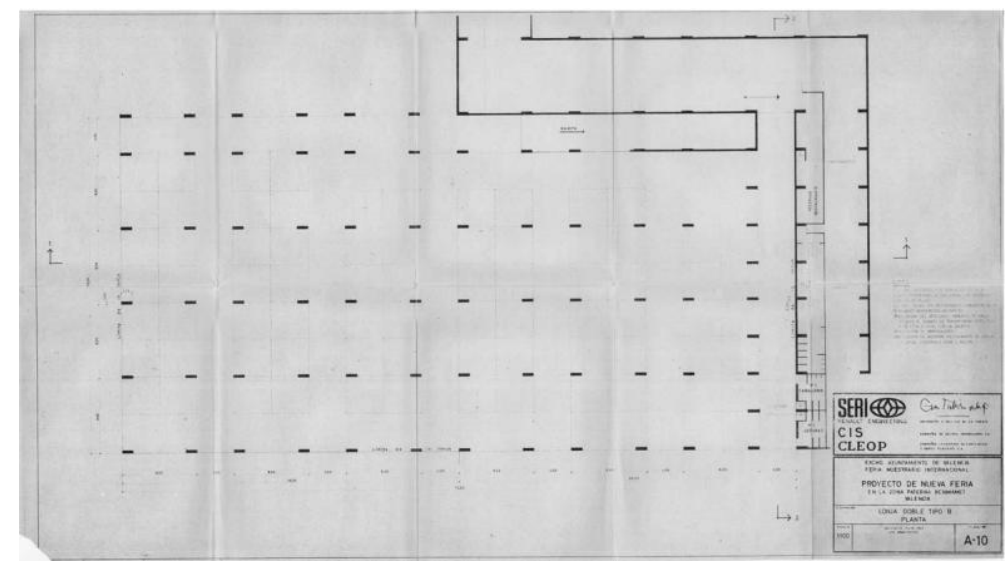

4. Planta de la lonja doble tipo B de la Feria de Valencia. Plano No A-10 (escala 1:100, Julio 1967).

Ésta nace de una unidad de construcción -la lonja de 36 × 36 m.- que se ajusta a la dimensión del módulo cuadrado de la parrilla y que básicamente corresponde a un espacio ordenado a partir de los pilares (pantallas) que sostienen un sistema de cubierta formado por una losa plegada en V, similar a la del Hospital de Venecia.

Cada lonja permite la creación de tres áreas de exposición de $8 \mathrm{~m}$. de anchura y $36 \mathrm{~m}$. de largo en las que las pantallas se disponen de tal forma que entre ellas se pueden organizar espacios expositivos basados en el módulo cuadrado de $4 \times 4 \mathrm{~m}$. Entre estas áreas de exposición se generan pasillos transversales -también de $4 \mathrm{~m}$. de ancho- que conectan con la circulación de borde de la lonja, integrándose al sistema de recorridos dentro de la Feria.

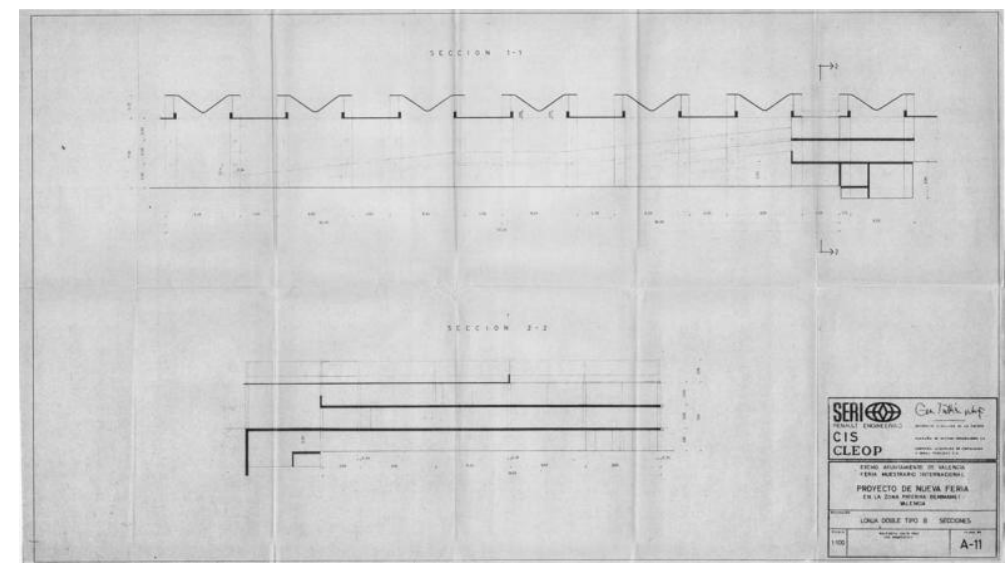

5. Secciones de la lonja doble tipo B de la Feria de Valencia. Plano No A-11 (escala 1:100, Julio 1967).

La pieza básica del proyecto se construye a partir de dos módulos contiguos de lonja, de los que uno de ellos resuelve con una rampa la diferencia de cota de nivel (2,50 m.) que conlleva la implantación de la trama sobre el

\footnotetext{
${ }^{14}$ Pérez de Arce, Rodrigo. Op. cit. p. 63. A este respecto debemos señalar que el binomio "arquitectura y juego" constituyese -para este autor- la primera de las seis claves temáticas que explican la obra de Jullian de la Fuente (ver pp. 20-47).
} 
terreno. Con esta pieza esencial del juego - en realidad una doble lonja- se produce el montaje de todo el conjunto de la Feria.

Comenzando en la cota inferior del terreno, el sistema semeja un dominó quebrado que va generando terrazas en zigzag. Estas plataformas se conectan entre sí mediante el sistema de rampas que, a su vez, generan ejes de circulación que las atraviesan en diagonal.

El juego con las diferentes alturas de las lonjas (5 m., 7,50 m. y 10 m.) permite -a nivel volumétrico- absorber las diferencias de cota entre las distintas plataformas, de manera que, combinando esas alturas, se consigue que la volumetría del conjunto de pabellones sólo presente un único escalonamiento para un interior que se desarrolla en cuatro plataformas con desniveles de 2,50 m. entre ellas. La edificación adquiere así un carácter horizontal, a pesar de las grandes diferencias topográficas.

En la zona superior del terreno se localiza la entrada general que sirve también al área de congresos, restaurante y hotel ${ }^{15}$, que forman una unidad dentro del proyecto. Su arquitectura se plantea siguiendo el mismo sistema estructural de los pabellones de exposición y constituye un magnífico ejemplo de integración de diferentes programas dentro de una misma construcción.

Con una pieza -la lonja-formalmente similar a la estructura de la unité de soins del Hospital de Venecia, Jullian crea un maravilloso juego de dominó que se va adaptando a las curvas de nivel del terreno para completar un mosaico bajo el que se cobija un espacio continuo pero diverso en el que tiene lugar la confluencia de las gentes.

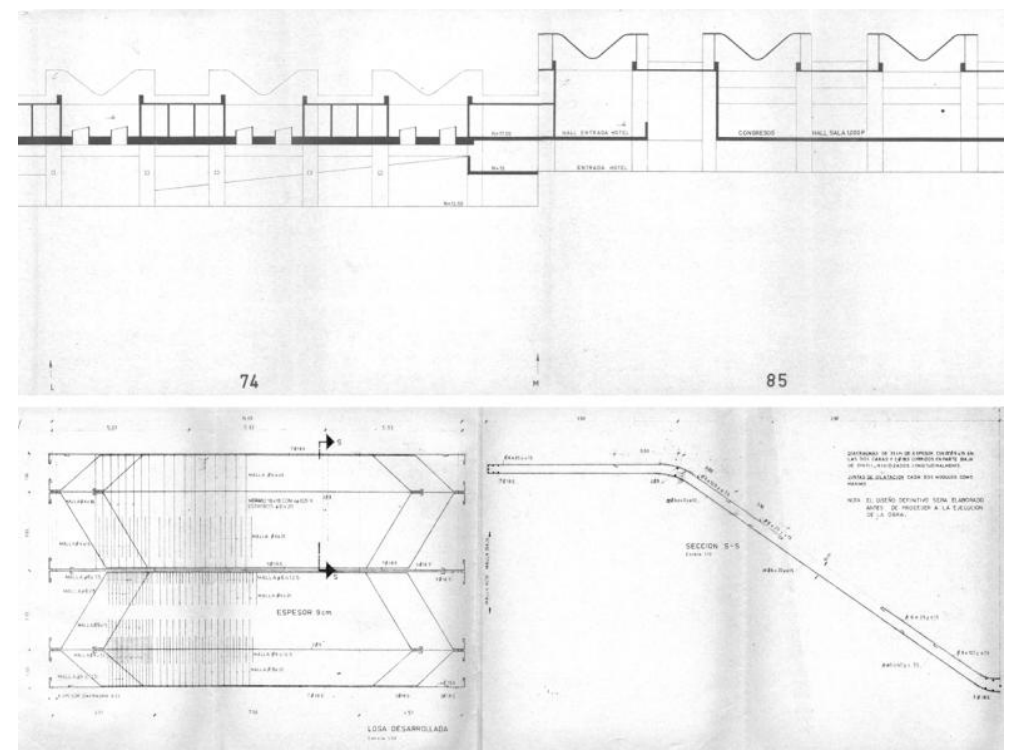

6. Detalle de la sección longitudinal 5, hotel y sala de congresos, Plano No A-26 (escala 1:100, Julio 1967). Armado de la losa en forma de V de 16 metros del proyecto original, Plano $\mathrm{N}^{\mathrm{o}} \mathrm{E}-17$ (escala 1:50 y 1:10, Julio 1967).

\footnotetext{
${ }^{15}$ Este conjunto estaba previsto en una segunda fase de construcción que no llegó a realizarse.
} 


\section{Apuntes para una conclusión}

La seriedad, que no frivolidad, del juego, no debe ocultar un hecho al que no es ajeno nuestra ponencia: la Feria de Valencia lamentablemente ha desaparecido. Sólo un pequeño pabellón sobrevive absolutamente descontextualizado y sin protección patrimonial. Si desaparece, lo habrá hecho la única edificación en la que se ensayó a gran escala la construcción de las cubiertas del Hospital de Venecia ${ }^{16}$.

Se da la paradoja de que ambas obras - coetáneas en su desarrollo proyectual- sólo permanecerán en la memoria de la arquitectura moderna, así como en sus documentos, pero no como obra construida en la que se pueda experimentar la percepción del espacio, su escala, su luminosidad, el ambiente: la emoción de una arquitectura viva.

Como memoria, cabe destacar esa íntima relación que tiene el proyecto de Valencia con el del Hospital veneciano. Ambos pioneros y coetáneos respecto de aquel concepto arquitectónico del Mat-Building que, con evidente protagonismo de Shadrach Woods, había surgido en las reuniones del Team 10. Paradigma que -como ya hemos señalado- pondrá en valor Alison Smithson en su famoso artículo de $1974^{17}$, y de cuya filosofía proyectual se han realizado posteriormente diversos ejemplos.

A este respecto debemos señalar el artículo de Timothy Hyde "How to Construct an Architectural Genealogy", en el que, con una mirada hacia delante, hace un recorrido por la arquitectura moderna vista desde la óptica del Mat-Building. Los ejemplos destacado por Hyde van desde el barrio de Tuscolano de Libera (1951-54), hasta la propuesta de Reiser, Unemoto y Eisenman para Nueva York (1999). Un itinerario en el que -a diferencia de Smithson- el autor se detiene en el proyecto de Jullian para la Feria de Valencia: "from the Crystal Palace to Tange's plan for Osaka, provide further training in mat building... multiple programs and extensive circulation contained by comprehensive structure permit flexible use and movement within a legible system"18.

Cabe recordar que Jullian escribe a los Smithson en septiembre de 1.967 informándoles del inicio del proyecto de la embajada francesa en Brasilia y anticipándoles que les mostrará el proyecto de la Feria de Valencia, cuyos planos de desarrollo sabemos que están fechados en julio de ese año. También informa del proyecto a Mónica Pidgeon, editora de Architectural Design, en diciembre de ese mismo año ${ }^{19}$. Sin embargo la primera vez que aparece publicado el proyecto es con motivo del artículo, que en 1968, le dedica Giuseppe Mazzariol en la revista Lotus; cuya portada está protagonizada por un maravilloso boceto de la sección. Allí la Feria es presentada como el primer proyecto que -tras la muerte del maestro- acomete en solitario Jullian de la Fuente, su último hijo espiritual. El proyecto es contemplado, por Mazzariol, bajo el paradigma de un moderno y gigantesco "Bazar" en el que la experiencia de un recorrido no obligatorio y diverso adquiere todo el protagonismo ${ }^{20}$.

\footnotetext{
${ }^{16}$ Antes de la construcción de la Feria, Jullian ensayó, a pequeña escala, la construcción de este tipo de cubierta en un ático para oficinas en Lyon. Ver: Pérez de Arce, Rodrigo. Op. cit. pp. 114-115.

${ }^{17}$ Smithson, Alison. Op. cit.

${ }^{18}$ Hyde, Timothy. "How to Construct an Architectural Genealogy". En: Sarkis, Hashim (Ed.). Le Corbusier's Venice Hospital and the Mat Building Revival. p. 108

${ }^{19}$ La Facultad de Arquitectura, Diseño y Estudios Urbanos (FADEU) de la Pontificia Universidad Católica de Chile custodia actualmente el Fondo Documental Guillermo Jullian de la Fuente. La carta a los Smithson así como la enviada a Mónica Pigdeon tienen los códigos FGJ-C0269 y FGJ-C0278 respectivamente. Dichos documentos sólo puede ser consultados en la biblioteca de la FADEU. Conocemos su contenido por las fichas del catálogo redactadas por la archivista Paloma Parrini.

${ }^{20}$ Mazzariol, Giuseppe. "La Feria di Valencia di Guillermo Jullian”. En: Lotus. No 5. 1968. p 35.
} 
También la idea del recorrido preside el apartado que Pérez de Arce dedica a la Feria en su artículo de Massilia 2007. Bajo el encabezamiento "Valencia y los laberintos funcionales", el autor se detiene en aquellos diagramas topológicos que constituyen la primera aproximación conceptual al proyecto y en los que, como vimos, la idea de "juego" adquiría un papel destacado: "fuertemente polarizada, la idea de recorrido que emerge de estos esquemas parece querer iluminar los tránsitos y relaciones entre recintos y, al mismo tiempo, estimular la deriva de los pasos perdidos, operación que recuerda ciertos juegos figurativos" ${ }^{21}$.

Un importante apunte sobre esta presencia del juego en la obra de Jullian, lo encontramos en el comentario de Pablo Allard contenido dentro de su artículo "Bridge over Venice". Ensayo en el que queda clara esa cercanía entre el proyecto para Valencia y el Hospital veneciano. Hablando de cómo entiende Jullian el orden, dice Allard: "this incorporation of a dimension of play in constant dialogue with working rigor is more serious than its superficial reading, and it has been a persistent characteristic of Jullian's work"22.

Por último, Giuliano Gresleri, considera en su artículo: "La Chiesa in collina e l'atelier tra cielo e mare", que la Feria de Valencia constituye el desarrollo de los procedimientos agregativos del Hospital de Venecia y señala cómo el proyecto, a pesar de ser poco conocido, encontrará repercusión en la búsqueda arquitectónica de los años sucesivos; donde la idea de un espacio capaz de crecer armónicamente sin interrumpir la concepción espacial del módulo básico que lo compone, será recogida por el Atelier 5 y por Herman Hertzberger e influirá en el trabajo de muchos otros arquitectos ${ }^{23}$.

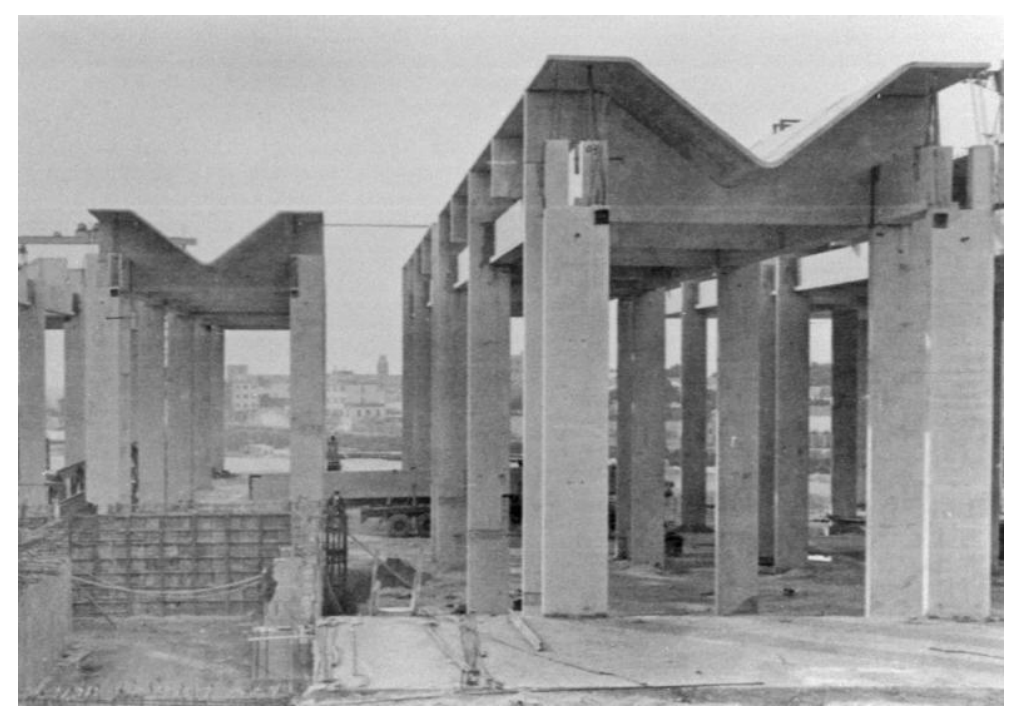

7. Construcción de las losas en V en Feria de Valencia.

\footnotetext{
${ }^{21}$ Pérez de Arce Antoncic, Rodrigo. "Entre el atajo y la promenade: recorridos en la obra de Guillermo Jullian". En: Massilia. 2007. p. 146. El autor ilustra esta idea con una lámina que recoge una poesía laberíntica cúbica dedicada al obispo de Oporto (c. 1600).

${ }^{22}$ Allard, Pablo. "Bridge over Venice. Speculations on Cross-fertilization of Ideas between Team 10 and Le Corbusier (after a Conversation with Guillermo Jullian de la Fuente)". En: Sarkis, Hashim (Ed.). Le Corbusier's Venice Hospital and the Mat Building Revival. pp. 18-35.

${ }^{23}$ Gresleri, Giuliano: "La Chiesa in collina e l'atelier tra cielo e mare”. En: Massilia. 2007. p. 40.
} 


\subsection{Aproximación a una cronología}

El proyecto para el Hospital de Venecia y el de la Feria de Valencia discurren por caminos muy diferentes. Mientras que el Hospital avanza siguiendo una línea continua, clara y documentada, la Feria transita por una ruta discontinua, borrosa y olvidada. De ahí que cuando estos caminos se acerquen lo hagan a veces en terrenos en los que se desdibujan las huellas que nos permiten reconocer el momento exacto de esa aproximación.

1917 señala, en la arquitectura del Corbu, la época que va de las Casas "Dom-ino" a las Casas "Citrohan". Pero en Valencia es el año en el que nace una institución que se convertirá con el tiempo en uno de los grandes instrumentos de proyección internacional de la ciudad: la Feria de Valencia.

Sin embargo, 1917 sólo constituye una anécdota dentro de esta cronología: la de situar en el horizonte la fecha de 1967 como la de la celebración del cincuenta aniversario del nacimiento de aquella institución. No es extraño pues, que en el ánimo de los promotores del nuevo recinto ferial estuviera la idea de señalar, con una gran obra de arquitectura, esa conmemoración ${ }^{24}$. Nuestra aproximación a esta fecha tan significativa requiere, por tanto, un gran salto en el tiempo.

1963

17 de diciembre. Le Corbusier escribe a Carlo Ottolenghi -presidente de los Ospedali Civili Riuniti di Veneziaaceptando hacerse cargo del proyecto de Hospital de Venecia y comprometiéndose a entregar una primera fase del mismo en mayo del año siguiente.

Es también el año de la fundación de la Feria Internacional del Mueble de Valencia: Fernando Mateu de Ros es su director y fundador.

1964

El Ayuntamiento de Valencia se hace con los terrenos del antiguo campo de tiro de Paterna -situado a las afueras de la ciudad- que serán destinados a albergar el nuevo recinto ferial.

1965

30 de marzo. La Embajada de España en París dirige una carta a Le Corbusier en la que le comunican el interés de una persona en España por entrevistarse con el maestro para: "exposer un projet espagnol d'urbanisation de la banlieue de Valence ${ }^{25}$. El Agregado Cultural de la Embajada -Rafael F. Quintanilla- firma el documento. Le Corbusier incorporada a la carta una nota manuscrita que no hemos podido descifrar. Del contenido de la misiva se deduce que el maestro -aunque desconocía los detalles- estaba informado sobre el asunto.

1 de abril. La Secretaria de Le Corbusier remite una carta al Agregado Cultural de la Embajada española, en la que le comunica que el maestro "sera heureux de vous recevoir en mai ainsi que la personne venant d'Espagne qui doit lui donner les précisions utiles relatives à votre projet d'urbanisation. Toutefois il vous demande de bien vouloir le prévenir assez longtemps à l'avance de la date de ce rendez-vous car il doit s'absenter de Paris à plusieurs reprises" ${ }^{26}$.

\footnotetext{
${ }^{24}$ La feria se celebraba entonces en el entorno de Viveros, en pleno centro de la ciudad.

${ }^{25}$ Fundación Le Corbusier. Documento No 12-16-251.

${ }^{26}$ Fundación Le Corbusier. Documento No 12-16-252.
} 
8 de abril. Le Corbusier y Jullian de la Fuente viajan a Venecia para la entrega del proyecto del Hospital.

Mayo. Es el mes previsto para la cita con Le Corbusier. Sabemos -por testimonios familiares directos- que Fernando Mateu de Ros y Carlos Soria -arquitecto director de los servicios técnicos de Feria Muestrario de Valencia- viajaron a París para entrevistarse con el maestro. Sin embargo no es posible confirmar la fecha exacta de esa visita ya que no se han encontrado, por el momento, los documentos que la acrediten.

Agosto. Durante todo el mes Jullian está en Venecia trabajando en el proyecto del Hospital: concretando quien debe hacer los cálculos de la estructura de hormigón y quien debe pagarlos. El 27 de ese mes muere Le Corbusier en Cap Martin.

En el primer consejo de administración del hospital tras la muerte del Corbu se decide que sea su colaborador Jullian de la Fuente quien continúe con el proyecto en los términos contractuales.

Otoño-invierno. Se cierra el Atelier de la rue de Sèvres y Jullian, Oubrerie, Jeannette -la secretaria de Le Corbusier- y Andreini -el contable del Atelier- se trasladan a un pequeño estudio de pintor en la rue Daguerre del barrio de Montparnasse, que había pertenecido a Shadrach Woods.

1966

19 de febrero. Con motivo de la inauguración de la V Feria del Juguete de Valencia se presenta a la prensa la maqueta y planos de las nuevas instalaciones feriales. Se trata de un anteproyecto firmado por Carlos Soria y Román Giménez. El diario Levante lo recoge con estas palabras: "Los correspondientes proyectos han sido realizados por los arquitectos Carlos Soria y don Ramón [sic] Giménez. Ocuparán una superficie de unos cien mil metros cuadrados, y su coste se estima en unos seiscientos millones de pesetas... El proyecto es magnifico, ya que ofrece una espléndida perspectiva y amplios espacios para el montaje de estas ferias comerciales"27.

28 de febrero. Jullian entrega en Venecia el proyecto titulado "H.VEN.LC - Variante à 800 lits" que lleva el sello de Le Corbusier.

1966-[1967]

Se abandona el proyecto de Soria y Giménez a favor del realizado por la ingeniería francesa SERI-Renault, para la que Jullian trabajaba como arquitecto asesor tras la muerte del $\mathrm{Corbu}^{28}$.

1967

Febrero-junio. El Atelier Jullian de la rue Daguerre trabaja en el proyecto para la Feria de Valencia (F VAL). Intervienen: Oubrerie, Andreini, Gabillar, Domeyko, Gambarin, Koszel y Jampen.

\footnotetext{
${ }^{27}$ Levante. Domingo, 20 de febrero de 1966. p. 7. El proyecto es muy diferente al realizado posteriormente por Jullian de la Fuente: un sistema de pabellones independientes se ordenan en torno a lo que parece ser una gran avenida central que actúa como eje de simetría que desemboca en un pabellón o plaza semicircular.

${ }^{28}$ En un reportaje publicado en El Mercantil Valenciano (Domingo, 30 de junio de 2002. p. 5), Román Jiménez comenta que fueron los problemas para financiar la obra los que dieron al traste con aquella propuesta y declara que fue SERI-Renault la que aportó la solución para el dinero, pero traían bajo el brazo su propio proyecto.
} 
Julio. SERI-Renault (Arquitecto: Jullian de la Fuente): "Proyecto de Nueva Feria en la zona de Paterna Benimàmet. Valencia".

Septiembre. El hijo de Carlos Soria se incorpora al Atelier Jullian de la rue Daguerre para colaborar en el proyecto de la Embajada de Francia en Brasilia, que Jullian había heredado del Corbu ${ }^{29}$.

1968

Dan comienzo las obras de la Feria de Valencia con el movimiento de tierras.

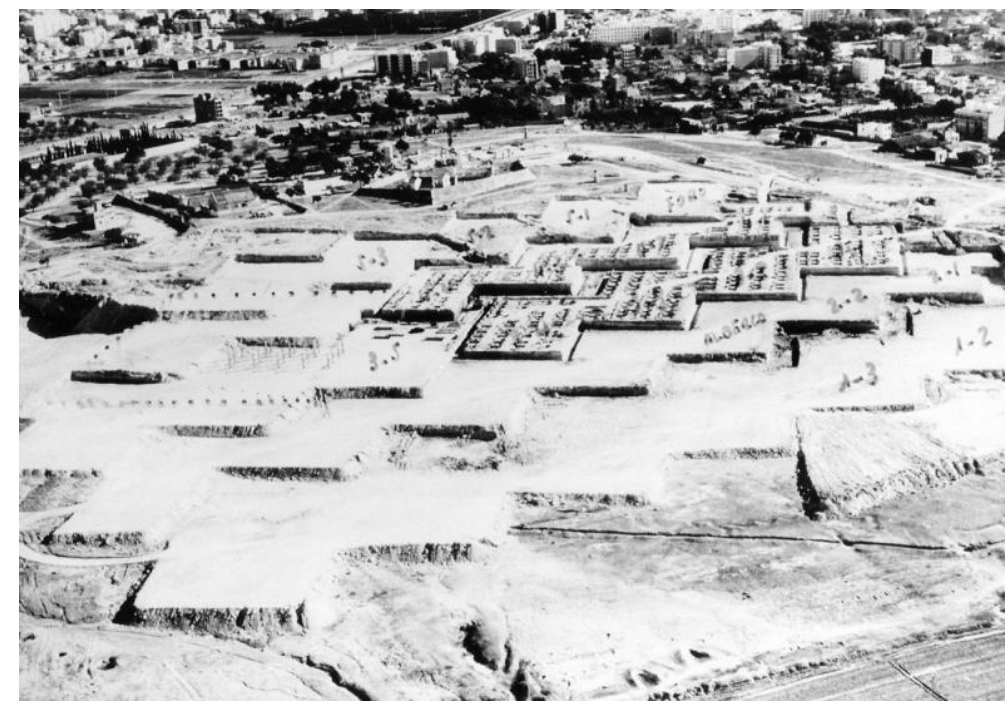

8. Explanación y aterrazamiento de los terrenos en los que se edificará Feria de Valencia.

1969

La construcción de la Feria de Valencia sigue adelante. El diario Levante recoge la noticia de la visita a las obras del alcalde de la ciudad con estas palabras: "Como técnico asesor de las autoridades figuraba el arquitecto municipal, director de las obras, don Román Giménez, coautor, con el arquitecto don Carlos Soria del proyecto de esta nueva Feria que se está levantando, desarrollando una idea del arquitecto francés Jullian de la Fuente, discípulo y seguidor del célebre Le Courbusier [sic]"30. La primera fase de edificación será inaugurada en octubre de este año.

\footnotetext{
${ }^{29}$ Destacamos este hecho para acercarnos al modo de trabajar del Atelier de la rue Daguerre. En conversación con Carlos Soria Giner (mayo-2015) sabemos que su llegada a París -tras haber acabado sus estudios de Arquitectura en la escuela de Barcelona- coincidió con la obra de reforma de aquel pequeño local. Tres días antes se había marchado Mario Botta, quien había estado trabajando durante un año en el desarrollo del proyecto para Venecia. Según su testimonio directo parece ser que Giuseppe Samoná estaba interesado en que en el Hospital interviniesen los hermanos Petrilli -alumnos suyos en la Escuela de Arquitectura de Venecia- por lo que Carlos dedicó su labor al proyecto de la Embajada. Soria Giner, comenta el hecho de que Guillermo Jullian y José Oubrerie heredaron el método de trabajo de Le Corbusier, así como los proyectos que estaban en marcha a la muerte del maestro: donde lo dibujado por el Corbu se mantenía hasta el final, mientras que en lo nuevo se permitían cierta flexibilidad. Recuerda que en el Atelier trabajaban con papel sulfurizado y dibujaban los detalles a escala 1:1 en hojas de papel continuo que se colgaban de las paredes. Posteriormente, el trabajo pasaba a la ingeniería SERI-Renault que se encargaba de realizar el proyecto de ejecución.

${ }^{30}$ Levante. Sábado, 12 de enero de 1969. p. 11.
} 


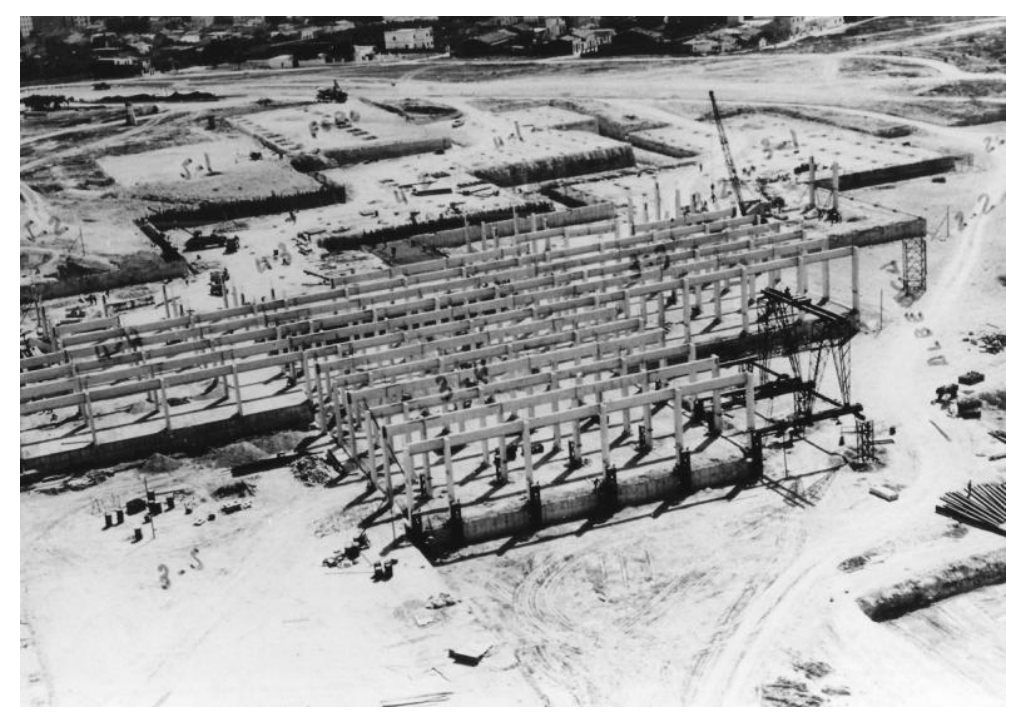

9. Construcción de la estructura de las lonjas de Feria de Valencia.

1970

Se abandona definitivamente la construcción del Hospital de Venecia, días antes de esa decisión se habían tomado las primeras muestras para la cimentación de los pilotis ${ }^{31}$.

La Feria de Valencia comienza su andadura. Casi treinta años después el edificio será demolido.

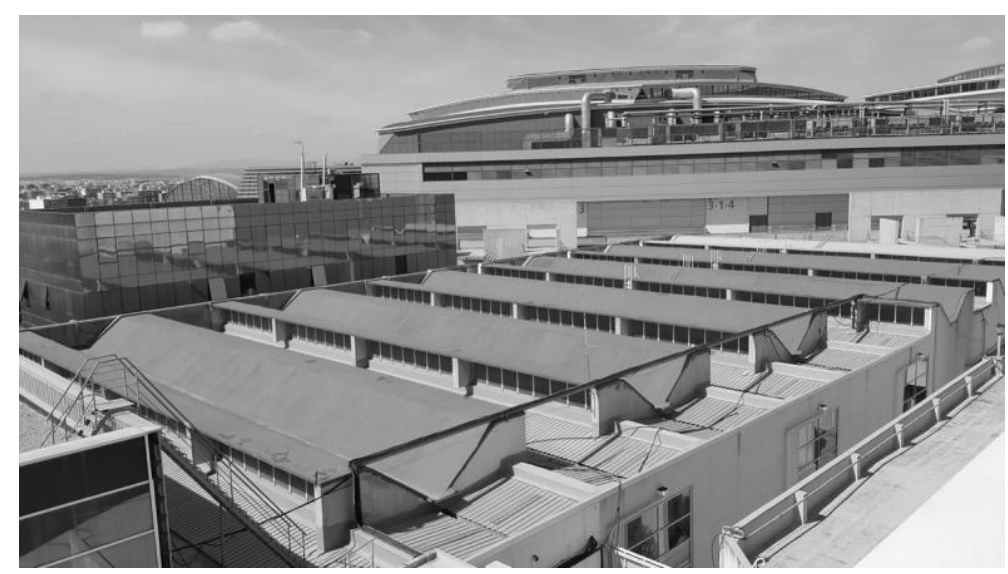

10. Cubierta del Foro Norte, única lonja existente.

\subsection{La Feria de Valencia: valor patrimonial}

A pesar de lo poco que queda del edificio y su absoluta descontextualización, entendemos el valor patrimonial que tiene la permanencia de la Feria Valencia de Jullian de la Fuente en la memoria arquitectónica de la ciudad. Quedan los documentos orales, escritos y gráficos que completan el recuerdo de haber transitado por sus lonjas sin la justa valoración de su importancia en la historia de la arquitectura moderna.

${ }^{31}$ Allard, Pablo. Op. cit. p. 27. 


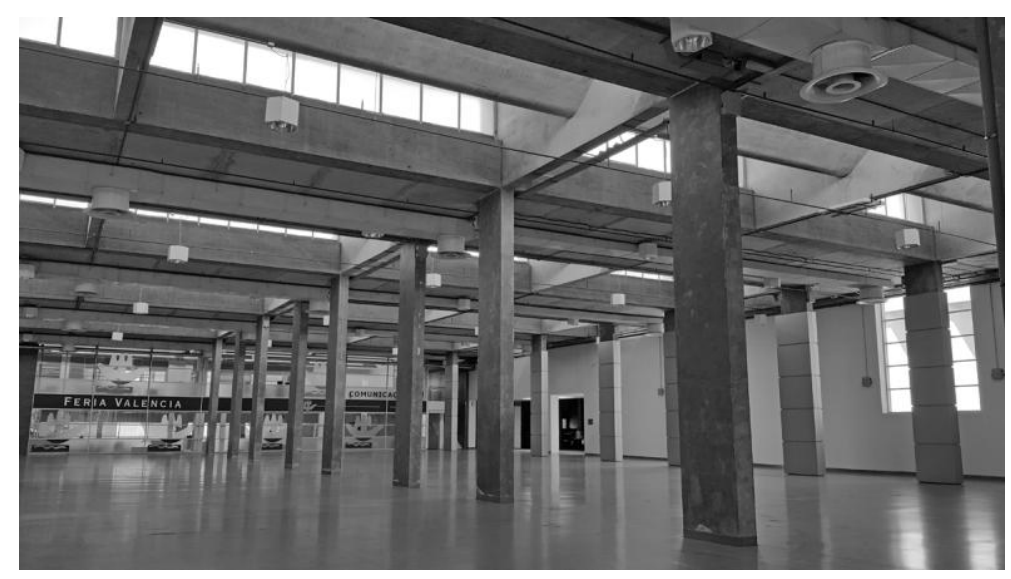

11. Espacio interior del Foro Norte.

Hay de inmediato que conseguir poner en valor lo poco que se conserva del conjunto. A la protección del bien inmueble hay que unir la protección de toda la documentación existente, la cual sabemos que está correctamente archivada y documentada en los originales depositados en la Facultad de Arquitectura, Diseño y Estudios Urbanos de la Pontificia Universidad Católica de Chile. Así como añadir una labor informativa sobre la íntima relación que existe entre el hecho compositivo recogido en el Mat-Building y los proyectos del Hospital de Venecia y la Feria de Valencia. Deber informativo del que el presente trabajo constituye un primer intento.

Es igualmente necesario proponer la inclusión del módulo de lonja que aún permanece en pie, dentro de una ficha de catálogo del DOCOMOMO IBERICO (Documentation and Conservation of buildings, sites and neighbourhoods of the Modern Movement- Sección Ibérica), ya que entendemos que es un registro abierto, y que -aunque en su primera fase se incluyeron los 166 edificios considerados más representativos del período comprendido entre 1.925 y 1.965 - se ha ido ampliando con obras coetáneas a la Feria de Valencia: como el edificio de la Confederación Hidrográfica del Júcar en Valencia (1.962-1.970) de Miguel Colomina, o las obras de Fernando Moreno Barberá de la Universidad Laboral de Cheste (1.965-1.969), la Facultad de Derecho (1.9591.968) y la de Filosofía y Letras (1.960-1.970) ambas en Valencia. Ficha que se documentaría con la descripción del proyecto original, el estado actual y los valores arquitectónicos de la pieza construida, como son: la fabricación in situ, el uso de la luz cenital, la materialidad del hormigón y el orden compositivo. Con una referencia clara como obra reconocida del Atelier de Le Corbusier e inscrita en el concepto Mat-Building.

Proponemos también la inclusión del módulo de lonja en el Catálogo de Espacios y Bienes Protegidos del Plan General de Ordenación Urbana de Valencia, con la categoría de Bien de Interés Cultural. Su inclusión en el Inventario General de Patrimonio Cultural Valenciano, según dispone en su artículo 15 la Ley 5/2.007 de Patrimonio Cultural Valenciano.

Esta protección y catalogación llevaría aparejada, pues, la colaboración entre administraciones públicas y, en este caso las instituciones locales - concretamente el Ayuntamiento de Valencia y Feria Valencia- para que adopten las medidas recogidas en la citada Ley en cuanto a conservación, protección, difusión y posibilidad de conocer el citado Bien. 


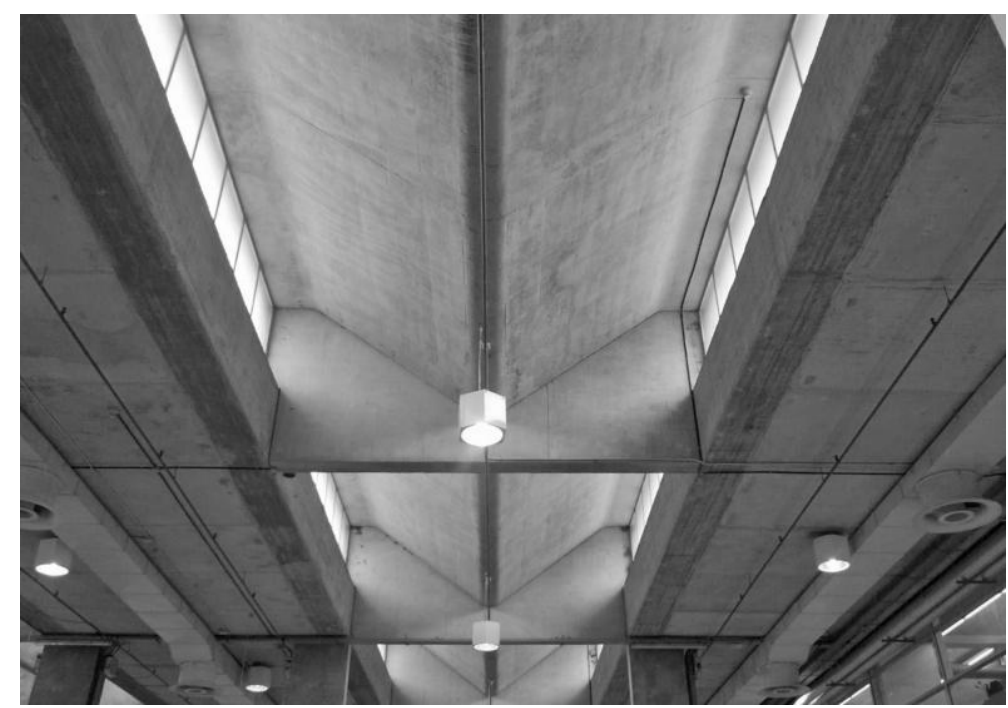

12. Detalle del lucernario, losa en V del Foro Norte.

\section{Conclusión}

Si algo queda claro en nuestra aproximación a la Feria de Valencia es la conexión directa de este proyecto con Le Corbusier y su Atelier.

En primer lugar porque el trabajo constituye, en realidad, una obra colectiva realizada -tras la muerte del maestro- por sus propios dibujantes con su Chef d'Atelier al frente. De la lectura del índice de bocetos y planos depositados en el Fondo Documental Guillermo Jullian de la Fuente, de la Pontificia Universidad Católica de Chile, se observa que muchos de ellos llevan la mano de Jullian, de José Oubrerie, Fernando Domeyko, Amedeo Petrilli y otros colaboradores del Atelier ${ }^{32}$.

En segundo lugar porque el proyecto para Valencia se superpone en el tiempo al desarrollo del Hospital de Venecia, que estaba a cargo de los mismos proyectistas. Una superposición que, no obstante, trasciende ese propio plano temporal para conectarse con los aspectos puramente arquitectónicos que vinculan ambas arquitecturas.

En tercer lugar porque de todos los proyectos que quedaron sobre las mesas de dibujo del Atelier de la rue de Sèvres tras la muerte de Le Corbusier; o bien no siguieron adelante -como en el caso del Museo del siglo XX en Nanterre- o bien se transformaron radicalmente por exigencias del promotor -como sucedió con la Embajada de Francia en Brasilia- o bien -como en el caso la iglesia de Firminy- se terminaron mucho tiempo después, pero siguiendo las ideas esbozadas por el Corbu. De manera qué, el único proyecto que podemos considerar de "nueva planta", pero conectado directamente con los modos de pensar y hacer de Le Corbusier, es la Feria de Valencia.

No podemos terminar esta investigación sin hacer referencia a un dato que no puede ser pasado por alto: la buena sintonía que debió establecerse entre Carlos Soria -arquitecto director de las obras- y Jullian de la Fuente, a raíz

\footnotetext{
${ }^{32}$ Conocemos estos datos por las fichas del catálogo redactadas por la archivista de la FADEU Paloma Parrini.
} 
del trabajo conjunto en la construcción de la Feria. Una afinidad que, sin duda, contribuyó a que en Valencia se hiciera realidad la construcción de una obra lecorbuseriana. Prueba de esta sintonía la tenemos en el dibujo que Jullian regala a Soria y que, aunque alejado del concepto arquitectónico que inspira el proyecto para Valencia, nos devuelve a aquel paradigma en el que nos hemos apoyado para articular nuestro análisis: la idea de juego en la composición arquitectónica.

Se trata de un boceto del alzado de la Casa del Hombre de Zurich (1963), que lleva las iniciales L C y la fecha 4/4/63. En el dibujo, dos elementos de cubierta -uno cóncavo y el otro convexo- se articulan entre sí para componer un paraguas bajo el que se cobija una construcción independiente ${ }^{33}$. Maravilloso ejemplo de ese modo de entender la arquitectura, como el juego sabio correcto y magnífico de los volúmenes reunidos bajo la luz.

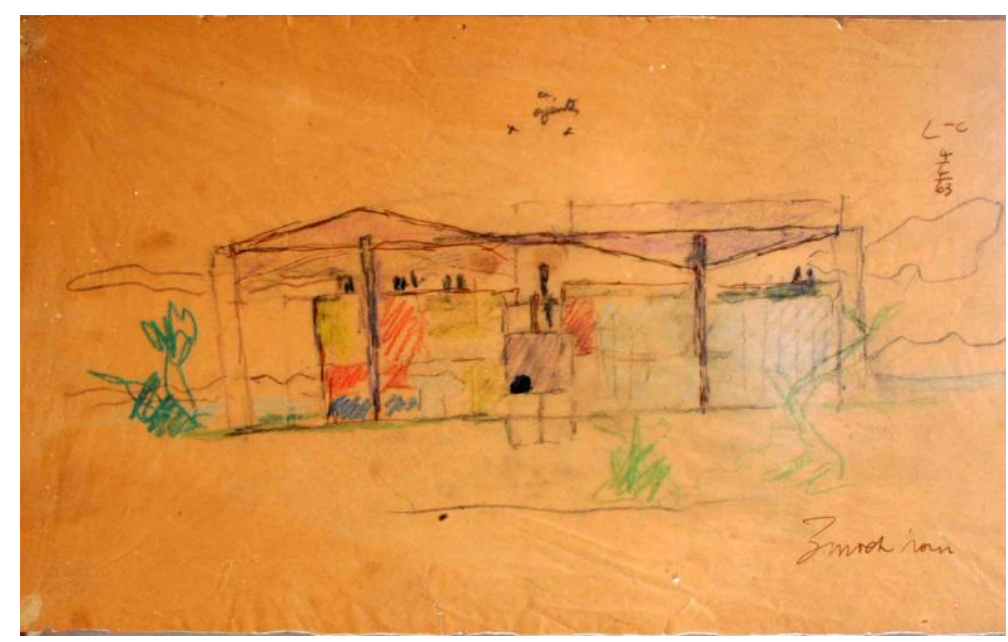

13. Boceto "Casa del Hombre" L-C 4/4/63, propiedad particular.

\section{Epílogo}

En un texto revisado a lo largo del tiempo y reescrito por última vez en julio de 1965 (Mise au point), Le Corbusier se refiere a la seriedad del juego y de los que juegan con estas palabras: "Il n'y a que ceux qui jouent qui soient des types sérieux! ... Les alpinistes, les rugbymen et les joueurs de cartes, et les joueurs de roulette sont des fumistes, car ils ne jouent pas ....”34.

Una seriedad que sujeta el juego a la disciplina de la regla, convertida así en la propia razón de su existencia. Y por eso añade: "Oui, la règle c'est le jeu ... Tout à l'intérieur de la règle! Rien hors de la règle! Sinon je n'ai plus de raison d'exister. Là est la clef. Raison d'exister: jouer le jeu"35.

\footnotetext{
${ }^{33}$ El dibujo lo conserva Mariano Soria Giner, quién nos ha facilitado su fotografía y otorgado el permiso para su publicación.

${ }^{34}$ Le Corbusier. Mise au point. En: Žaknić, Ivan. The Final Testament of Père Corbu. New Haven - Londres: Yale University Press, 1997. p. 114.

${ }^{35}$ Ibid. Existe traducción autorizada de este texto realizada por Jorge Torres, quién en el ensayo que la acompaña repasa algunas otras referencias a la idea del juego y sus reglas en diferentes textos del maestro. A este respecto ver: Torres, Jorge. Pensar la Arquitectura: "Mise au point" de Le Corbusier. pp. 91-92.
} 


\section{Agradecimientos}

Queremos agradecer a la Institución Ferial de Valencia así como al Archivo histórico del Ayuntamiento de Valencia las facilidades que nos han dado para acometer esta investigación. También expresamos nuestro agradecimiento a la familia de Fernando Mateu de Ros y, en especial, a sus hijos Fernando e Ignacio y a su nieto Fernando que tan amablemente nos han atendido. Asimismo expresamos nuestro agradecimiento a los hijos de Carlos Soria: Carlos, Mariano y Juan Luis Soria Giner por sus aportaciones a este trabajo, así como a la documentalista de la FADEU de la Pontificia Universidad Católica de Chile Paloma Parrini y a Delphine DS. Studer de la Fundación Le Corbusier. Por último agradecemos la ayuda que nos han prestado Tomás Roselló (arquitecto técnico e investigador) y nuestros compañeros José Luis Esparza y José Luis Ros.

\section{Procedencia de las imágenes}

Imagen_1. Autores de la ponencia: plano canónico de la Feria de Valencia.

Imagen_2. Feria de Valencia: vista de la maqueta.

Imagen_3. Fundación Le Corbusier : H VEN LC. Plano No 6286: "Unité de bâtisse - 4 Unités de soins" (FLC 28236) OFLC-ADAGP.

Imagen_4. Feria de Valencia: Planta lonja doble tipo B de la Feria de Valencia.

Imagen_5. Feria de Valencia: Secciones lonja doble tipo B de la Feria de Valencia.

Imagen_6. Feria de Valencia: Detalle sección longitudinal 5, hotel y sala de congresos. Armado de losa en forma de $\mathrm{V}$ de 16 metros.

Imagen_7. Feria de Valencia: Construcción de las losas en V.

Imagen_8. Feria de Valencia: Explanación y aterrazado de los terrenos.

Imagen_9. Feria de Valencia: Construcción de la estructura de las lonjas.

Imagen_10. Autores de la ponencia: Cubierta del Foro Norte, única lonja existente.

Imagen_11. Autores de la ponencia: Espacio interior del Foro Norte.

Imagen_12. Autores de la ponencia: Detalle del lucernario, losa en V del Foro Norte.

Imagen_13. Mariano Soria: Boceto "Casa del Hombre" L-C 4/4/63.

\section{Bibliografía}

Allard, Pablo. "Bridge over Venice. Speculations on Cross-fertilization of Ideas between Team 10 and Le Corbusier (after a Conversation with Guillermo Jullian de la Fuente)". En: Sarkis, Hashim (Ed.). Le Corbusier's Venice Hospital and the Mat Building Revival. Múnich - Londres - Nueva York: Prestel, 2001. pp. 18-35.

Colquhoun, Alan. "Interacciones formales y funcionales. Un estudio de dos de los últimos proyectos de Le Corbusier". En: Arquitectura moderna y cambio histórico: ensayos 1962-1976. Barcelona: Gustavo Gili, 1978. pp. 34-44.

Gresleri, Giuliano: “La Chiesa in collina e l'atelier tra cielo e mare”. En: Massilia. 2007. pp. 32-42.

Huizinga, Johan. Homo ludens. Madrid: Alianza-Emecé, $6^{\mathrm{a}}$ reimp., 2007.

Hyde, Timothy. "How to Construct an Architectural Genealogy". En: Sarkis, Hashim (Ed.). Le Corbusier's Venice Hospital and the Mat Building Revival. Múnich - Londres - Nueva York: Prestel, 2001. pp. 104-117.

Le Corbusier. Mise au point. En: Žaknić, Ivan. The Final Testament of Père Corbu. New Haven - Londres: Yale University Press, 1997. pp. 141-155.

Mazzariol, Giuseppe. “La Feria di Valencia di Guillermo Jullian”. En: Lotus. No 5. 1968. pp. 34-59. 
O’Byrne Orozco, María Cecilia. El proyecto para el Hospital de Venecia de Le Corbusier. Director: Josep Quetglas. Universidad Politécnica de Cataluña - Escuela Técnica Superior de Arquitectura de Barcelona, 2007.

Pérez de Arce Antoncic, Rodrigo. "Entre el atajo y la promenade: recorridos en la obra de Guillermo Jullian". En: Massilia. 2007. pp. 138-153.

Pérez de Arce, Rodrigo. Guillermo Jullian. Obra abierta. Santiago de Chile: ediciones ARQ-Pontificia Universidad Católica de Chile, 2000.

Pérez Oyarzún, Fernando. "Valparaíso y los años formativos”. En: Massilia 2007, pp. 54-67.

Petrilli, Amedeo. Il testamento di Le Corbusier: il progetto per l'Ospedale di Venezia. Venecia: Marsilio, 1999.

Smithson, Alison. "How to Recognise and Read Mat-Building: Mainstream Architecture as It Has Developed towards the Mat-Building". En: Sarkis, Hashim (Ed.). Le Corbusier's Venice Hospital and the Mat Building Revival. Múnich - Londres - Nueva York: Prestel, 2001. pp. 90-103.

Torres, Jorge. Pensar la Arquitectura: "Mise au point" de Le Corbusier. Madrid, Abada, 2014.

Vásquez, Claudio. "Conversación con Guillermo Jullian de la Fuente”. En: Massilia. 2007. pp. 18-31. 